\title{
Recurrent Conjunctival Squamous Cell Carcinoma and Intraocular Tumor Extension after Topical Erythropoietin: A Case Report
}

\author{
Sepehr Feizi ${ }^{a}$ Hamed Esfandiarib \\ aOphthalmic Research Center, Department of Ophthalmology, Shahid Beheshti University

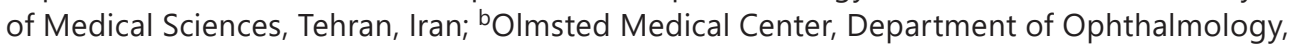 \\ Rochester, MN, USA
}

Keywords

Conjunctival squamous cell carcinoma - Intraocular extension · Topical erythropoietin

\begin{abstract}
Topical erythropoietin has been recently introduced for the treatment of avascular conjunctival and scleral lesions. Before this treatment can be routinely used, however, its safety profile and contraindications should be determined. Herein, we report a case of recurrent conjunctival squamous cell carcinoma (SCC) and intraocular tumor extension after treatment with topical erythropoietin for excisional biopsy-induced scleral necrosis. An 87-year-old man underwent excisional biopsy for a conjunctival leukoplakic mass. Histological examination showed a well-differentiated SCC on the postoperative day 10. All borders of the specimen were reported to be involved with tumoral cells. The patient did not receive further surgical intervention or topical mitomycin since he developed surgically induced scleral necrosis on the postoperative day 14 . Topical erythropoietin $3,000 \mathrm{IU} / \mathrm{mL}$ was started every $6 \mathrm{~h}$, and avascular scleral lesion healed over 21 days of treatment with topical erythropoietin. However, 4 months after complete improvement, the tumor recurred with extension into the anterior chamber. Ultrasound biomicroscopy showed the involvement of sclera, iris root, and ciliary body with blunting of the anterior chamber angle. Orbital extension was not detected in magnetic resonance imaging. Topical erythropoietin administered in eyes with a history of conjunctival SCC could be linked to tumor recurrence and intraocular invasion. We recommend avoiding topical erythropoietin in eyes with existing conjunctival SCC or a previous history of conjunctival SCC that was incompletely removed.
\end{abstract}

\section{Karger ${ }^{\prime \prime}=$}




\section{Introduction}

Ocular surface squamous neoplasia (OSSN) describes neoplastic lesions of corneal and conjunctival epithelium with dysplastic changes ranging from intraepithelial neoplasia to invasive squamous cell carcinoma (SCC). OSSN is the most common nonpigmented cancer of the cornea and conjunctiva with a reported incidence varying from 0.13 to 1.9 per 100,000 people [1]. OSSN typically occurs in the interpalpebral zone of the bulbar conjunctiva, originating at the mitotically active limbal region and often spreads onto the cornea. Its risk factors include exposure to ultraviolet light and chemicals, human immunodeficiency virus or papilloma virus infection, immunosuppression caused by medications, and cigarette smoking [2, 3].

Surgical excision with wide tumor-free conjunctival margins, using a no-touch technique, is the time-honored therapy for conjunctival OSSN, often with adjunctive cryotherapy [4]. Other recently introduced treatment modalities include mitomycin-C, 5 -fluorouracil, and interferon alpha- $2 \mathrm{~b}[5,6]$. Recurrences after treatment of OSSN are common, ranging from $5 \%$ to $56 \%$ [7]. Majority of the recurrences are diagnosed during the first year of treatment; however, SCC may recur even after 5 years of follow-up [7]. Several factors are associated with higher risk of recurrence including increased age, large tumor size, tarsal location, xeroderma pigmentosa, stand-alone surgical excision with no adjunctive therapy, higher malignancy grade, and positive surgical margins at histopathologic examination [8-10].

Erythropoietin, a glycoprotein hormone, regulates hematopoiesis by stimulating the differentiation and proliferation of hematopoietic stem cells and inhibiting their apoptosis [11]. Erythropoietin and its receptor are localized in different tissues such as vascular endothelium and retina, indicating that this hormone is important for maintaining their physiological homeostasis [11]. Recently, we demonstrated the efficacy of erythropoietin in the management of scleral necrosis caused by different etiologies including ocular procedures, chemical burns, and collagen vascular disease $[12,13]$. This glycoprotein hormone can effectively improve avascular conjunctival and scleral lesions by inducing vascularization and reducing the inflammatory response and apoptosis [14]. Before topical erythropoietin can be routinely used for the treatment of scleral necrosis, its safety profile and contraindications should be determined. Herein, we present a case report of recurrent SCC with intraocular tumor extension in a patient who was treated with topical erythropoietin for excisional biopsy-induced scleral necrosis.

\section{Case Presentation}

An 87-year-old man presented with a 3-month history of foreign body sensation, redness, and a progressively enlarging mass in his right eye. His past history was remarkable for pterygium removal surgery in the right eye, performed 3 years ago, and uncomplicated phacoemulsification and intraocular lens implantation in both eyes, performed 10 years ago. In addition, he had a history of chronic lymphocytic leukemia that was treated with systemic chemotherapy.

Best corrected visual acuity was 20/40 OD and 20/50 OS. Slit-lamp examination revealed a well-circumscribed elevated leukoplakic mass on the nasal bulbar conjunctiva in the interpalpebral area, measuring $5.5 \times 4.0 \mathrm{~mm}$ and sparing the cornea. Conjunctival congestion and feeding vessels were noted adjacent to the lesion. Other ocular examinations were unremarkable, and the left eye was normal. No regional lymphadenopathy was detected. An excisional biopsy with 3-mm margin was performed for presumed OSSN. Lamellar sclerectomy was not performed, and the patient did not receive intraoperative cryotherapy or antimetabolites. Postoperatively, the patient received topical antibiotic and betamethasone $0.1 \%$ every $6 \mathrm{~h}$, as well as frequent lubrication.

\section{Karger'}




\section{Case Reports in Ophthalmology}
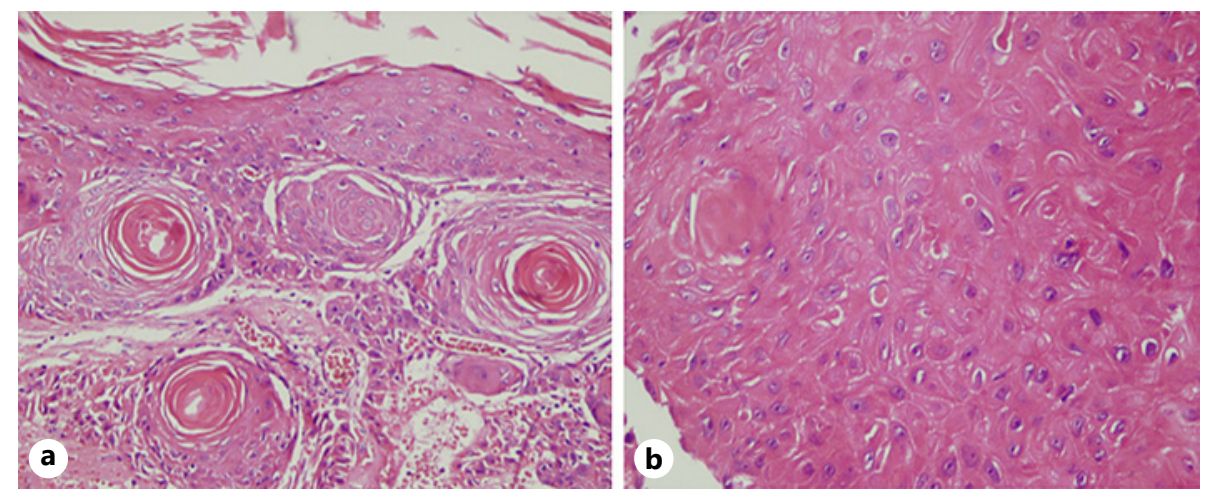

Fig. 1. Histopathologic examination shows well-differentiated SCC of the conjunctiva. a The microsection contains nests of irregularly arranged neoplastic squamous epithelial cells, originating from a keratinized squamous epithelium, keratin pearls, and mild lymphoplasmacytic infiltration interspersed between the tumor islands (hematoxylin $\&$ eosin stain; $\times 2$ magnification). b The neoplastic cells have abundant eosinophilic cytoplasm and large vesicular nuclei with distinct nucleoli (hematoxylin \& eosin stain; $\times 4$ magnification).
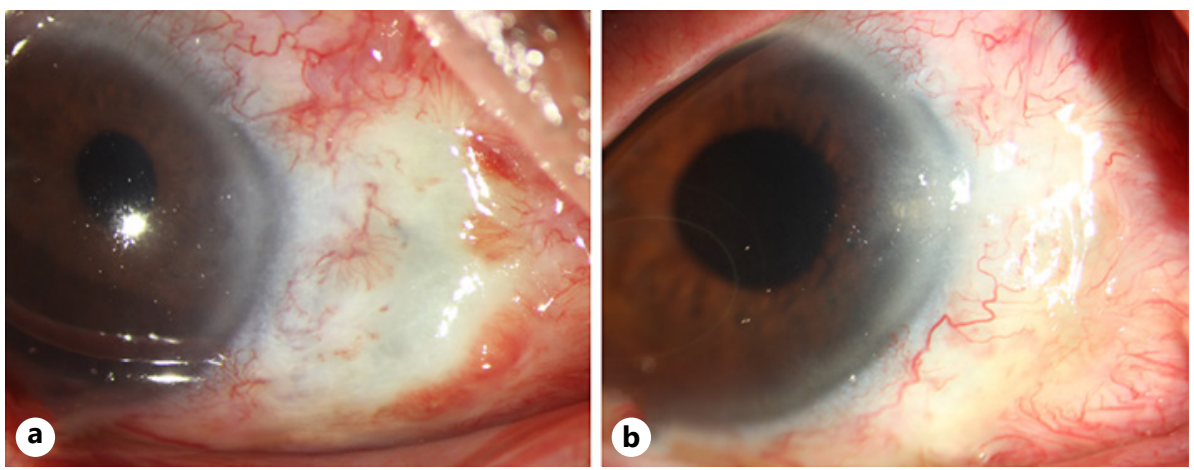

Fig. 2. a An area of scleral necrosis and melt developed 14 days after excisional biopsy of the conjunctival SCC. b Treatment with topical erythropoietin $(3,000 \mathrm{IU} / \mathrm{mL}$ four times a day $)$ resulted in epithelialization and vascularization of the avascular area after 21 days.

On the postoperative day 10 , histological examination disclosed nests of irregularly arranged neoplastic squamous epithelial cells, originating from a keratinized squamous epithelium and breaching the epithelium basement membrane (Fig. 1a). Other findings included keratin pearls and mild lymphoplasmacytic infiltration interspersed between the tumor islands (Fig. 1a). The neoplastic cells contained abundant eosinophilic cytoplasm and large vesicular nuclei with distinct nucleoli consistent with well-differentiated invasive SCC (Fig. 1b). The biopsy specimen exhibited no spindle cell or mucoepidermoid characteristics. All borders of the specimen were reported to be involved with tumoral cells. Despite incomplete tumor removal, further surgical excision or adjuvant treatment was not performed.

On the postoperative day 14, the patient presented with chief complaint of injection and discomfort in his right eye. Slit-lamp examination disclosed avascular scleral necrosis and thinning in the site of tumor excision, measuring $7.5 \times 3.5 \mathrm{~mm}$. The surrounding conjunctiva and sclera were edematous with dilated superficial and deep vessels (Fig. 2a). The patient was diagnosed with surgically induced scleral necrosis. Therefore, topical betamethasone was discontinued; whereas, topical antibiotic and frequent lubrication were continued. Topical erythropoietin $(3,000 \mathrm{IU} / \mathrm{mL})$ was prepared by diluting $1.5 \mathrm{~mL}$ of commercially available 


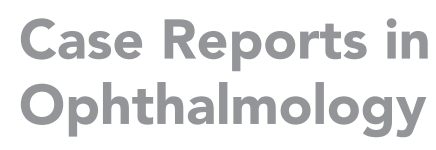

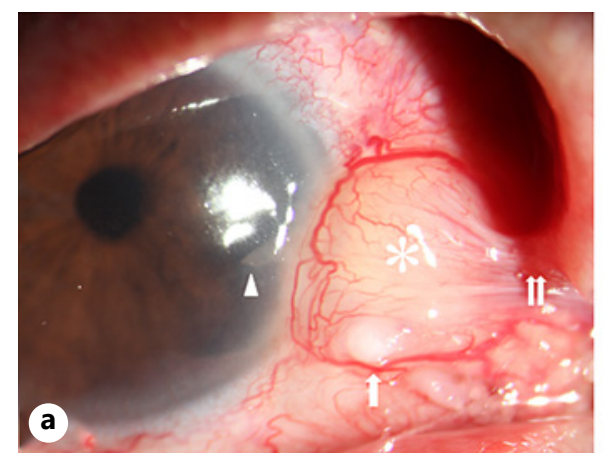

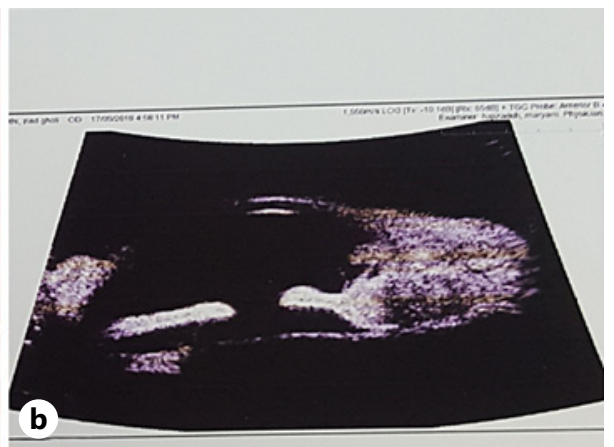

Fig. 3. a Four months after complete improvement of scleral necrosis, slit-lamp examination exhibits conjunctival bulge (asterisk) with areas of keratin (arrow) and symblepharon formation (double arrow) in the nasal quadrant. A white mass (arrowhead) is evident in the anterior chamber angle indicating intraocular tumor extension. b Ultrasound biomicroscopy shows hyperechoic corneoscleral thickening, moderately reflective ciliary body and iris mass with translucent areas, and blunting of the anterior chamber angle.

recombinant human erythropoietin solution for intravenous use in normal saline (PDpoetin, 10,000 IU/0.5 mL; Pooyesh Darou Biopharmaceutical Co., Tehran, Iran). This medication was started every $6 \mathrm{~h}$ and continued for 21 days until the avascular scleral lesion was completely epithelialized and vascularized (Fig. 2b). Systemic workup did not reveal human immunodeficiency virus infection, collagen vascular disease, rheumatoid arthritis, or related conditions.

Four months after complete improvement of the avascular scleral lesion, slit-lamp examination revealed conjunctival bulge with areas of keratin and symblepharon formation in the nasal quadrant (Fig. 3a). There was a white mass in the anterior chamber angle that had no connection with the previous cataract surgery incision (Fig. 3a). Dilated fundus examination was unremarkable. Ultrasound biomicroscopy showed hyperechoic corneoscleral thickening, moderately reflective ciliary body and iris mass with translucent areas, and blunting of the anterior chamber angle (Fig. 3b). Orbital extension of the tumor was not detected in contrast enhanced magnetic resonance imaging. The assessment of regional lymph nodes and systemic workup did not indicate systemic metastasis. The patient was diagnosed with recurrent SCC of the conjunctiva with intraocular tumor extension and was scheduled for enucleation. However, considering his systemic condition, he decided not to follow with enucleation.

\section{Discussion}

Conjunctival SCC is a slow-growing tumor that usually has a benign course; however, it can extend into the eye in $1-15 \%$ of cases, or even rarely metastasize $(<1 \%)[15,16]$. Intraocular involvement can manifest as corneal stromal opacification, glaucoma, chronic intraocular inflammation, and iris, ciliary body, and anterior chamber mass [15]. Risk factors for intraocular extension include nodulo-ulcerative pattern of the tumor, spindle cell, mucoepidermoid and poorly differentiated variants of carcinoma, tumor recurrence with history of prior tumor excision, and immunosuppression due to human immunodeficiency virus and medications $[15,17,18]$. Approximately, $80 \%$ of reported cases with intraocular extension of conjunctival SCC underwent excisional biopsy before the diagnosis of intraocular invasion $[15,19]$. It has been proposed that excisional biopsy could change the tumor or its surrounding in favor of more invasive tumor in case of recurrence [20]. The mean interval from tumor excision to intraocular tumor invasion is 7 months, ranging from $<1$ month to 35 months [15]. Another risk factor for intraocular extension of conjunctival SCC is previous or concurrent intraocular

\section{Karger's}


surgeries such as cataract extraction, penetrating keratoplasty, and glaucoma filtering surgery $[15,18]$. These procedures disrupt the integrity of the Bowman layer and create a tract for intraocular tumor extension. In addition, tumor can invade into the eye through scleral infiltration or along the tract of the anterior ciliary vessels $[15,18]$.

There were several risk factors for tumor recurrence and intraocular extension in our case such as increased age, incomplete excision of the tumor without adjunctive cryotherapy, a background of previous immunosuppression, and previous cataract surgery. While tumor removal was not complete, since the patient developed scleral necrosis, topical mitomycin, 5 -fluorouracil, or interferon alpha-2b was not started postoperatively. We believe that there was an association between tumor recurrence and topical erythropoietin administered for excisional biopsy-induced scleral necrosis. Surgically induced scleral necrosis is a rare but well-known complication after any type of ocular procedure, which can lead to devastating complications such as scleral infection and perforation. Therefore, early treatment of this complication is crucial to improve the prognosis. Free conjunctival autograft is an acceptable surgical intervention for this complication that can effectively cover the avascular scleral areas. This option, however, was not appropriate for our case as any attempts to cover the avascular scleral bed could obscure the signs of tumor recurrence. Recently, we reported the efficacy of topical erythropoietin for the treatment of avascular scleral lesions due to different etiologies such as ocular surgical procedures $[12,13]$. The results of our experimental study showed that topical erythropoietin induces vascularization of the necrotic sclera and reduces the inflammatory response and apoptosis [14]. After a thorough discussion with the patient, we decided on topical erythropoietin, which resulted in a complete improvement of the avascular scleral lesion. However, the patient returned with recurrence and intraocular extension of the conjunctival SCC. Increasing evidence have demonstrated that exogenous erythropoietin can enhance the growth of both erythropoietin receptor-positive and -negative cancer cells in patients with lymphoid, non-small cell lung, breast, head and neck, endometrial, cervical, gastric, and colon cancers [21-23]. This hormone may promote the growth and viability of tumors by enhancing the numbers and self-renewing capacity of cancer-initiating cells, inhibiting apoptosis, and stimulating angiogenesis $[23,24]$. It has been shown that blocking erythropoietin receptor can stop the growth and progression of tumor cells and reduce its capillary network which supports erythropoietin's role in tumor progression and proliferation [25]. Despite the presence of several other risk factors, our report suggests an association between topically administered erythropoietin and conjunctival SCC recurrence and intraocular extension.

Several ocular and systemic contraindications have been described for erythropoietin administration, including the presence of any hematological and solid malignancy, history of thrombi-embolic events and epilepsy, polycythemia, high systemic blood pressure, iris and retinal neovascularization, and proliferative diabetic retinopathy [11]. This case report along with previous studies suggests that topical erythropoietin administered in eyes with a history of conjunctival SCC can increase the risk of tumor recurrence with intraocular extension. Therefore, we recommend avoiding topical erythropoietin in eyes with existing conjunctival SCC or a previous history of conjunctival SCC.

\section{Statement of Ethics}

The patient gave his written informed consent to publish the case (including publication of images). This study protocol was reviewed and approved (approval number, SBMU 140030204) by the Ethics Committee of the Ophthalmic Research Center, affiliated with the Shahid Beheshti University of Medical Sciences in Tehran, Iran.

\section{Karger'}




\section{Conflict of Interest Statement}

The authors have no conflicts of interest to declare.

\section{Funding Sources}

The authors have no funding sources to disclose.

\section{Author Contributions}

Sepehr Feizi made substantial contribution to the design of the work, the acquisition and interpretation of data, and drafting of the work. Hamed Esfandiari made substantial contribution to revising the work critically for important intellectual content and manuscript review and editing. Both authors agree to be accountable for all aspects of the work.

\section{Data Availability Statement}

All data generated and analyzed during this study are included in this article.

\section{References}

1 Shields CL, Demirci H, Karatza E, Shields JA. Clinical survey of 1643 melanocytic and nonmelanocytic conjunctival tumors. Ophthalmology. 2004;111:1747-54.

2 Shields CL, Ramasubramanian A, Mellen PL, Shields JA. Conjunctival squamous cell carcinoma arising in immunosuppressed patients (organ transplant, human immunodeficiency virus infection). Ophthalmology. 2011; 118:2133-7.e1.

3 Verma V, Shen D, Sieving PC, Chan CC. The role of infectious agents in the etiology of ocular adnexal neoplasia. Surv Ophthalmol. 2008;53:312-31.

4 Shields CL, Shields JA. Tumors of the conjunctiva and cornea. Indian J Ophthalmol. 2019;67:1930-48.

5 Frucht-Pery J, Rozenman Y, Pe'er J. Topical mitomycin-C for partially excised conjunctival squamous cell carcinoma. Ophthalmology. 2002;109:548-52.

6 Boehm MD, Huang AJ. Treatment of recurrent corneal and conjunctival intraepithelial neoplasia with topical interferon alfa 2b. Ophthalmology. 2004;111:1755-61.

7 Miller CV, Wolf A, Klingenstein A, Decker C, Garip A, Kampik A, et al. Clinical outcome of advanced squamous cell carcinoma of the conjunctiva. Eye. 2014;28:962-7.

8 Galor A, Karp CL, Oellers P, Kao AA, Abdelaziz A, Feuer W, et al. Predictors of ocular surface squamous neoplasia recurrence after excisional surgery. Ophthalmology. 2012;119:1974-81.

9 Mirzayev I, Gündüz AK, Özalp Ateş FS, Özcan G, Işık MU. Factors affecting recurrence after surgical treatment in cases with ocular surface squamous neoplasia. Int J Ophthalmol. 2019;12:1426-31.

10 Li AS, Shih CY, Rosen L, Steiner A, Milman T, Udell IJ. Recurrence of ocular surface squamous neoplasia treated with excisional biopsy and cryotherapy. Am J Ophthalmol. 2015;160:213-9.e1.

11 Feizi S, Alemzadeh-Ansari M, Karimian F, Esfandiari H. Use of erythropoietin in ophthalmology: a review. Surv Ophthalmol. 2021. Epub ahead of print.

12 Feizi S, Javadi MA. Topical erythropoietin as a novel treatment for necrotizing scleritis after pterygium surgery: a pilot study. Cornea. 2021;40(8):1011-7.

13 Feizi S, Alemzadeh-Ansari M, Baradaran-Rafii A, Esfandiari H, Kheirkhah A. Topical erythropoietin for treatment of scleral necrosis. Ocul Immunol Inflamm. 2021. Epub ahead of print.

14 Feizi S, Kanavi MR, Safari S, Ebrahimi H, Javadi MA. Effects of topical erythropoietin on healing experimentallyinduced avascular scleral damage in a rabbit model. Exp Eye Res. 2020;190:107898.

15 Kaliki S, Jajapuram SD, Maniar A, Taneja S, Mishra DK. Ocular surface squamous neoplasia with intraocular tumour extension: a study of 23 patients. Eye. 2020;34:319-26.

16 Yousef YA, Finger PT. Squamous carcinoma and dysplasia of the conjunctiva and cornea: an analysis of 101 cases. Ophthalmology. 2012;119:233-40. 
17 Kamal S, Kaliki S, Mishra DK, Batra J, Naik MN. Ocular surface squamous neoplasia in 200 patients: a case-control study of immunosuppression resulting from human immunodeficiency virus versus immunocompetency. Ophthalmology. 2015;122:1688-94.

18 Murillo JC, Galor A, Wu MC, Kye NK, Wong J, Ahmed IO, et al. Intracorneal and intraocular invasion of ocular surface squamous neoplasia after intraocular surgery: report of two cases and review of the literature. Ocul Oncol Pathol. 2017;3:66-72.

19 McKelvie PA, Daniell M, McNab A, Loughnan M, Santamaria JD. Squamous cell carcinoma of the conjunctiva: a series of 26 cases. Br J Ophthalmol. 2002;86:168-73.

20 Erie JC, Campbell RJ, Liesegang TJ. Conjunctival and corneal intraepithelial and invasive neoplasia. Ophthalmology. 1986;93:176-83.

21 Leyland-Jones B. Breast cancer trial with erythropoietin terminated unexpectedly. Lancet Oncol. 2003;4:459-60.

22 Henke M, Laszig R, Rübe C, Schäfer U, Haase KD, Schilcher B, et al. Erythropoietin to treat head and neck cancer patients with anaemia undergoing radiotherapy: randomised, double-blind, placebo-controlled trial. Lancet. 2003;362:1255-60.

23 Annese T, Tamma R, Ruggieri S, Ribatti D. Erythropoietin in tumor angiogenesis. Exp Cell Res. 2019;374:266-73.

24 Phillips TM, Kim K, Vlashi E, McBride WH, Pajonk F. Effects of recombinant erythropoietin on breast cancerinitiating cells. Neoplasia. 2007;9:1122-9.

25 Ribatti D, Marzullo A, Gentile A, Longo V, Nico B, Vacca A, et al. Erythropoietin/erythropoietin-receptor system is involved in angiogenesis in human hepatocellular carcinoma. Histopathology. 2007;50:591-6. 